\title{
From Kerr-Newman Black Hole to Spinning Particle: Where is There Hidden the Dirac Equation?
}

\author{
Alexander Burinskii*i \\ Theor. Phys. Lab., Nuclear Safety Inst., Russian Academy of Sciences, Moscow, Russian \\ Federation \\ E-mail: burinskii@mail.ru
}

\begin{abstract}
Gravitational and electromagnetic fields of the over-rotating Kerr-Newman (KN) solution correspond to the fields of an electron. However, this metric has a topological defect, which creates the singular and two-sheeted space-time. This defect is regulated by a bubble-core of the KN solution, which is formed of the supersymmetric false-vacuum state of the Higgs field. The field model of this bubble has much in common with the famous MIT and SLAC bag models, but the model is geometrically inverted - the Higgs condensate is localized in the core, leaving undisturbed the external solution KN. By analysis of the consistent solutions of the Dirac equation, we obtain that two-sheeted Kerr-Schild geometry ensures the space-time implementation of the SM-concept on the originally massless leptons. Similar to other bag models, the spinning KN bag is deformed at rotations. For parameters of an electron it turns into a thin ellipsoidal disk, sharp border of which forms a ring-string similar to solitonic strings of the low enetrgy string theory. This string admits traveling waves, which deform surface of the bag and create a traveling singular pole, reproducing 'zitterbewegung' of the Dirac electron. The KN bag model combines the dressed and point-like electron in a single gravitating quark-string-bag complex, in which the point-like electron may be considered as a single quark confined by the bag, or as a singular end point of the ring-string.
\end{abstract}

Frontiers of Fundamental Physics 14

15-18 July 2014

Aix Marseille University (AMU) Saint-Charles Campus, Marseille, France

\footnotetext{
*Speaker.

${ }^{\dagger}$ This research is supported by the RFBR grant 13-01-00602.
} 


\section{1. introduction}

It has been discussed for long time that black holes $(\mathrm{BH})$ should be connected with elementary particles. However, the giant spin/mass and charge/mass ratios of the particles $\left(\mathrm{J} / \mathrm{m} \sim 10^{44}\right.$ and $e / m \sim 10^{22}$ in the dimensionless units $G=c=\hbar=1$ ) show that the black hole horizons should disappear, and the particles can only be associated with an over-rotating geometry $\mathrm{KN}$, without horizons. This space has gyromagnetic ratio $g=2$ as in the Dirac electron, and therefore the overrotating Kerr-Newman $(\mathrm{KN})$ solution really reproduces the gravitational and electromagnetic field of an electron. However, this space-time has a topological defect - the naked Kerr's singular ring, which is a branch line of space into two sheets: the sheet of advanced and sheet of the retarded fields. The Kerr-Schild form of metric [1]

$$
g_{\mu v}=\eta_{\mu v}+2 H k_{\mu} k_{v}, \quad H=\frac{m r-e^{2} / 2}{r^{2}+a^{2} \cos ^{2} \theta}
$$

in which $\eta_{\mu \nu}$ is metric of auxiliary Minkowski space $M^{4}$, (signature $(-+++)$ ), $H$ is a scalar function, $r$ and $\theta$ are ellipsoidal coordinates and $k_{\mu}$ is a null vector field, $k_{\mu} k^{\mu}=0$, forming a vortex polarization of Kerr space-time - the Principal Null Congruence (PNC) $\mathscr{K}$. . The surface $r=0$ represents a disklike "door" from negative sheet $r<0$ to positive one $r>0$. The smooth extension of the solution from retarded to advanced sheet (together with smooth extension of the Kerr PNC) occurs via disk $r=0$ spanned by the Kerr singular $\operatorname{ring} r=0, \cos \theta=0$ (see fig.1). The null vector fields $k^{\mu \pm}(x)$ turns out to be different on these sheets, and two different null congruences $\mathscr{K}^{ \pm}$, create two different metrics $g_{\mu \nu}^{ \pm}=\eta_{\mu v}+2 H k_{\mu}^{ \pm} k_{v}^{ \pm}$on the same Minkowski background.

The mysterious two-sheeted structure of the Kerr geometry caused searching diverse models for source of the $\mathrm{KN}$ solution avoiding negative sheet. Besides, singular metric conflicts with basic principles of quantum theory, which is settled on the flat space-time and negligible gravitation. Resolution of this conflict requires "regularization" of space-time, which has to be done before quantization, i.e. on the classical level. Singular region has to be excised together with negative sheet and replaced by a regular core with a flat internal metric $\eta_{\mu \nu}$. The internal metric should

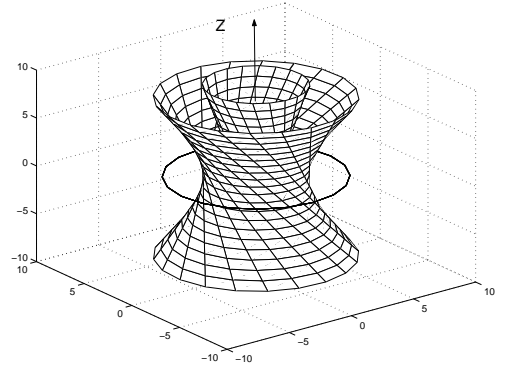

Figure 1: Kerr congruence forms vortex polarization of the Kerr space. Kerr singular ring is the branch line of Kerr space into two sheets. be matched with external KN solution. The consistent mathing was obtained by C. López (1984), who suggested the source of KN solution as a charged and rotating vacuum bubble, boundary of which $r=R$ is determined by the condition $H(r)=0$, or

$$
R=r_{e}=\frac{e^{2}}{2 m}
$$

Since $r$ is Kerr's ellipsoidal coordinate, the boundary of bubble covers the Kerr singular for $R>0$, and the bubble forms a thin rotating disk of the Compton radius $r_{c} \sim a=\hbar / m c$ and its thickness $R$ is equal to the known classical radius of electron $r_{e}=e^{2} / 2 m c$, so that the oblateness of the 
disk is equal to the fine structure constant $r_{e} / r_{c} \sim e^{2}=\alpha \sim 137^{-1}$. The bubble-source formed by López boundary was generalized in [3]) to a gravitating soliton model [2,3] which was build as a domain-wall bubble confining the Higgs field in a superconducting false-vacuum state.

Recently, [4] this model has been recognized as a gravitating bag model which has much in common with known MIT and SLAC bag models, [5, 6], however, it avoids the well-known conflict between Gravity and Quantum theory by dividing their spheres of influence. Region outside the disk-like bubble, $r>R$, is controlled by the classical KN solution. Near the boundary of the disk, $r \sim R$, gravity vanishes, and metric matches with flat metric inside the disk, where flatness of space is provided by false-vacuum state of the Higgs field $\Phi(x)$. The vacuum expectation value (vev) of this state $\sigma=<|\Phi|>$ is controlled by the Higgs mechanism of spontaneously broken symmetry. Thus, Quantum theory works in the flat space inside the bubble, while the classical KN gravity acts outside the Compton zone formed by the bubble boundary $R$. The Higgs condensate regulates also the KN electromagnetic (EM) field, pushing it from inside the bubble to domain wall boundary, which provides flatness of internal space required by quantum theory.

\section{Peculiarities of the phase transition for gravitating bag model}

The quartic potential $V(|\Phi|)=g\left(\bar{\sigma} \sigma-\eta^{2}\right)^{2}$ for self-interaction of the Higgs field is used in many nonperturbative models of the extended particles, and in particular, in the MIT and SLAC bag models. It creates the bag as a cavity (or bubble) in the Higgs condensate which fills all the external space, $r>R$. As a result, the gauge symmetry of the external gravitational and electromagnetic fields turns out to be broken. Gravitating bag model requires inverse situation: the Higgs condensate should be concentrated inside the bag, $r<R$, leaving the unbroken gauge symmetry in external space, $r>R$. This requires a more complex scheme of phase transition [2].

Supersymmetric phase transition requires three chiral fields $\Phi^{(i)}, i=1,2,3$. One of them, say $\Phi^{(1)}$, is identified as the Higgs field $\mathscr{H}$. The bag is formed of a domain wall, and the corresponding phase transition may be considered in a flat space-time. At this stage, we also ignore the EM field. We set new notations $(\mathscr{H}, Z, \Sigma) \equiv\left(\Phi^{1}, \Phi^{2}, \Phi^{3}\right)$. The required potential $V(r)=\sum_{i}\left|\partial_{i} W\right|^{2}$ is obtained by the known supersymmetric scheme [7] from the superpotential $W\left(\Phi^{i}, \bar{\Phi}^{i}\right)=Z(\Sigma \bar{\Sigma}-$ $\left.\eta^{2}\right)+(Z+\mu) \mathscr{H} \mathscr{\mathscr { H }}$, where $\mu$ and $\eta$ are real constants. The condition $\partial_{i} W=0$ determines two vacuum states separated by a positive spike of the potential $V$ in the transition zone $R-\delta<r<R-\delta$ :

(I) external vacuum, $r>R+\delta, V(r)=0$, with vanishing Higgs field $\mathscr{H}=0$, and

(II) internal false-vacuum, $r<R-\delta, V(r)=0$, with broken symmetry, $|\mathscr{H}|=\eta=$ const.

(III) intermediate region of the domain wall phase transition $R-\delta<r<R-\delta$.

Inside the bag the space is flat and the EM field vanishes, but gauge symmetry is broken. The Higgs condensate forms a supersymmetric superconducting false-vacuum state.

Transition zone (III). The space may still be considered as flat, but the Lagrangian includes interaction of the Higgs condensate with EM field. Field model of this type was used by NielsenOlesen for a vortex string in superconducting media [8],

$$
\mathscr{L}_{N O}=-\frac{1}{4} F_{\mu \nu} F^{\mu v}+\frac{1}{2}\left(\mathscr{D}_{\mu} \mathscr{H}\right)\left(\mathscr{D}^{\mu} \mathscr{H}\right)^{*}+V(|\mathscr{H}|),
$$

where $\mathscr{D}_{\mu}=\nabla_{\mu}+i e A_{\mu}$ is a covariant derivative, $F_{\mu \nu}=A_{v, \mu}-A_{\mu, \nu}$, and $\nabla_{\mu} \equiv \partial_{\mu}$ is reduced to derivative in flat space with a flat D'Alembertian $\partial_{v} \partial^{v}=\square$. For interaction of the complex Higgs 
field $\mathscr{H}(x)=|\mathscr{H}(x)| e^{i \chi(x)}$ with the Maxwell field we obtain the following complicated systems of the nonlinear differential equations

$$
\begin{aligned}
D_{v} D^{v} \mathscr{H} & =\partial_{\mathscr{H}} V, \\
\square A_{\mu}=I_{\mu} & =e|\mathscr{H}|^{2}\left(\chi, \mu+e A_{\mu}\right) .
\end{aligned}
$$

Inside the bag we should have $I_{\mu}=0$, and (2.3) yields two remarkable consequences, [2, 3]:

(A) The vortex of the KN vector potential $A_{\mu}$ forms a quantum Wilson loop placed along the border of the disk-like bag, which leads to quantization of the angular momentum,

(B) Phase of the Higgs field should compensate the component $A_{0}$ which leads to oscillations of the Higgs field with the frequency $\omega=2 m$.

\section{Fermionic sector}

The bag models give significant progress in the fermionic sector of the extended particle-like solutions. In the SLAC bag theory, [6], the Dirac equations interacting with the classical vev of the Higgs field $\sigma$-field take the form

$$
\left(i \gamma^{\mu} \partial_{\mu}-g \sigma\right) \psi=0
$$

where $g$ is a dimensionless coupling parameter. One sees that the Dirac field $\psi$ acquires from the Higgs field effective mass $m=g \sigma$, which takes maximal value $m=g \eta$ outside the bag and vanishes inside the bag. The quarks are confined, getting inside the bag a more favorable energetic position, and this is the principal idea of the confinement mechanism. For gravitating bag model, geometric position of the Higgs condensate is inverted "inside out" to have unbroken the gravitational and EM fields of the external KN solution. Thus, the Dirac equation will have nonzero mass $m=g \eta$ inside the bag, while outside the bag it will be massless. In the Weyl basis the Dirac equation splits into the left and right chiral equations for the spinor components of the Dirac bispinor $\Psi^{\dagger}=\left(\phi_{\alpha}, \bar{\chi}^{\dot{\alpha}}\right)$.

$$
\sigma_{\alpha \dot{\alpha}}^{\mu} i \partial_{\mu} \bar{\chi}^{\dot{\alpha}}=m \phi_{\alpha}, \quad \bar{\sigma}^{\mu \dot{\alpha} \alpha} i \partial_{\mu} \phi_{\alpha}=m \bar{\chi}^{\dot{\alpha}} .
$$

Outside the bag, $r>R-\delta$, the mass vanishes and these equations become independent

$$
\sigma_{\alpha \dot{\alpha}}^{\mu} i \partial_{\mu} \bar{\chi}^{\dot{\alpha}}=0, \quad \bar{\sigma}^{\mu \dot{\alpha} \alpha} i \partial_{\mu} \phi_{\alpha}=0 .
$$

They must be consistent with spinor structure of the external $\mathrm{KN}$ solution, which is determined by the Kerr theorem [4]. The KN solution may be represented in the Kerr-Schild (KS) form through the both Kerr congruences, the outgoing $k_{\mu}^{+}$or the ingoing $k_{\mu}^{-}$, but not the both simultaneously. Taking for the physical sheet of the KN solution the outgoing Kerr congruence $k_{\mu}^{+}$, corresponding to the retarded EM field and the metric $g_{\mu \nu}^{+}$, we obtain that the advanced Kerr congruence and EM field become inconsistent with this physical sheet. Being aligned with another congruence $k_{\mu}^{-}$, the advanced fields should be positioned on a separate sheet which different metric $g_{\mu \nu}^{-}$.

This problem disappears inside the bag, where $H=0$, the space is flat, $g^{ \pm}=\eta_{\mu v}$, and difference between these two metrics disappears. Due to consistency conditions the gravitational interaction drops out, and the Dirac equations take the ordinary flat space-time form. The consistency conditions $k^{\mu} \gamma_{\mu} \Psi=0$ turn into equations for eigenfunctions of the helicity operator

$$
(\mathbf{k} \cdot \sigma) \phi=\phi, \quad(\mathbf{k} \cdot \sigma) \chi=-\chi .
$$


One sees that the spinors $\phi$ and $\chi$ have opposite helicity, forming the "left-handed" $\phi$ and "righthanded" helicity states, aligned with the outgoing null direction $k_{\mu}^{+}=(1, \mathbf{k})$ and ingoing direction $k_{\mu}^{-}=(1,-\mathbf{k})$ correspondingly. The requirement of the consistency of the Dirac solutions with KN geometry enforces us to return the removed two-sheeted structure of the Kerr geometry, this time to restore it outside the KN source. Therefore, two massless Weyl spinors $\phi_{\alpha}$ and $\bar{\chi}^{\dot{\alpha}}$ should live on the different sheets of the external KN solution. In the flat space inside the bag, they are joined in a single Dirac bispinor and obtain mass from the Higgs field via Yukawa coupling. Two-sheeted structure of the Kerr-Schild geometry ensures the space-time realization of the SM-concept, in which the originally massless leptons acquire mass from the Higgs field.

Variation of the mass term on the regions of space is a new feature of the Dirac equation following from theory of the bag models. The Dirac wave function, solution of the Dirac equation with variable mass term, avoids the region with a large bare mass, and tends to get an energetically favorable position. In the SLAC bag model [6] this problem is solved by variational approach. The corresponding Hamiltonian is

$$
H(x)=\Psi^{\dagger}\left(\frac{1}{i} \vec{\alpha} \cdot \vec{\nabla}+g \beta \sigma\right) \Psi,
$$

and the energetically favorable wave function has to be determined by minimization of the averaged Hamiltonian $\mathscr{H}=\int d^{3} x H(x)$ under the normalization condition $\int d^{3} x \Psi^{\dagger}(x) \Psi(x)=1$. It yields

$$
\left(\frac{1}{i} \vec{\alpha} \cdot \vec{\nabla}+g \beta \sigma\right) \Psi=\mathscr{E} \Psi
$$

where $\mathscr{E}$ appears as the Lagrangian multiplier enforcing the normalization condition. Similar to results of the SLAC-bag model, one expects that the Dirac wave function will not penetrate deep in the region of large bare mass $m=g \eta$, and will concentrate in a narrow transition zone at the bag border $R-\delta<r<R+\delta$. As it motivated in [6], narrow concentration of the Dirac wave function is admissible for scalar potential which does not lead to the Klein paradox.

The exact solutions of this kind are known only for two-dimensional case, and the corresponding variational problem should apparently be solved numerically by using the ansatz $\tilde{\Psi}=f(x) \Psi(x)$, in which $f(x)$ is a variable factor for the Dirac solution based on the Weyl spinors $\phi_{\alpha}, \bar{\chi}^{\dot{\alpha}}$ consistent with the corresponding outgoing and ingoing Kerr congruences.

\section{Stringy deformations of bag: unification of the bare and dressed electron}

Taking the bag model conception, we should also accept the dynamical point of view that the bags are soft and may easily be deformed [6]. By deformations the bags may form stringy structures. The typical deformations of the bags are radial and rotational excitations forming the flux-tubes of the open strings. The old Dirac's model of an "extensible" spherical electron [9] may be considered as a prototype of the bag model with spherically symmetric radial excitations. The bag-like source of the KN solution without rotation, $a=0$, represents the Dirac model of a spherical "extensible" electron, which has in rest the classical electron radius $R=r_{e}=e^{2} / 2 m$. The $\mathrm{KN}$ rotating disk-like bag may be considered as the Dirac bag stretched by rotation to the disk of Compton radius, $a=\hbar / 2 m c$, which corresponds to the zone of vacuum polarization of a "dressed" electron. The degree of oblateness of the KN bag is close to $\alpha=137^{-1}$. Sharp boundary of this 
disk plays the role of a ring-string, which may be accompanied by traveling waves. Field structure of this string, [10], is similar to that of fundamental string, obtained by Sen solution to low energy string theory.

Boundary of the disk is very close to position of the Kerr singular ring, and regularization of the KN source gives to this string the cut-off parameter $R=r_{e},(1.2)$. In accord with [11,12] this string may carry the EM traveling waves which deform the bag surface. The EM excitations of the Kerr background [1] are defined by analytic function $A=\psi(Y, \tau) / P^{2}$ where $Y=e^{i \phi} \tan \frac{\theta}{2}$ is a complex projective angular variable, $\tau=t-r-i a \cos \theta$ is a complex retarded-time parameter and $P=2^{-1 / 2}(1+Y \bar{Y})$ for the Kerr geometry at rest. Vector potential is determined by function $\psi$ as follows [1]

$$
A_{\mu} d x^{\mu}=-\operatorname{Re}\left[\left(\frac{\psi}{r+i a \cos \theta}\right) e^{3}+\chi d \bar{Y}\right], \quad \chi=2 \int(1+Y \bar{Y})^{-2} \psi d Y
$$

The simplest function $\psi=-e$, corresponding to stationary $\mathrm{KN}$ solution, creates a frozen circular

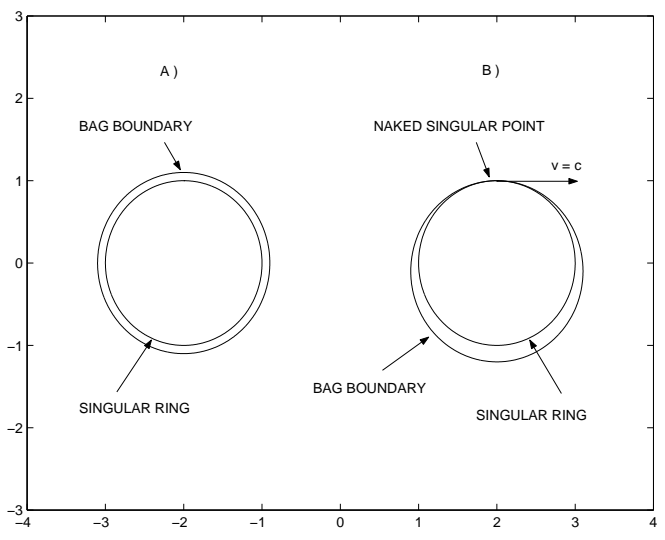

Figure 2: Cut-off parameter is determined by distance $R=r_{e}$ from the bag boundary to singular ring. A) Axially symmetric $\mathrm{KN}$ solution, $R=e^{2} / 2 m$. B) Deformation of the $\mathrm{KN}$ bag by stringy excitation.

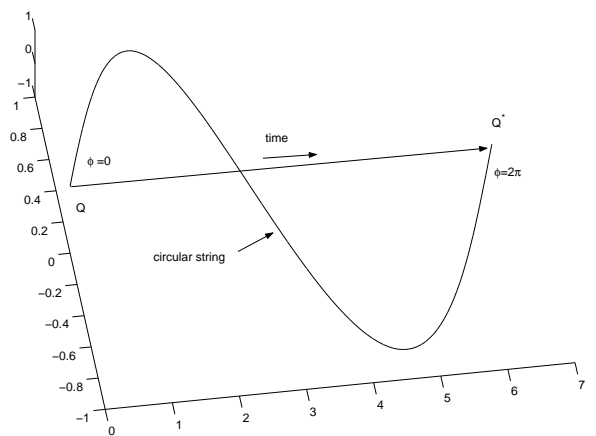

Figure 3: Circular light-like string propagating along the bag border joined with time-like zeromode of the KN solution at singular pole.

EM wave, which is locally plane and "propagates" along the Kerr singular ring. By regularization, it obtains the constant cut-off parameter $R=r_{e}$, see Fig.2.1A. It is a zero-mode excitation of the regularized Kerr string. Along with many other possible stringy waves, interesting effect shows the simple wave solutions

$$
\psi=e\left(1+\frac{1}{Y} e^{i \omega \tau}\right) .
$$

Function $\psi$ acts on the metric through the function $H$

$$
H=\frac{m r-|\psi|^{2} / 2}{r^{2}+a^{2} \cos ^{2} \theta} .
$$

As we have seen in sec.2, the condition $H=0$ determines the boundary of disk $R=|\psi|^{2} / 2 m$, which acts as the cut-off parameter for EM field. One sees that solution (4.2) takes in equatorial plane $\cos \theta=0$ the form $\psi=e\left(1+e^{-i(\phi-\omega t)}\right)$, and the cut-off parameter $R=|\psi|^{2} / 2 m=\frac{e^{2}}{m}(1+$ 
$\cos (\phi-\omega t)$ depends on $\phi-\omega t$. Vanishing $R$ at $\phi=\omega t$ creates singular pole which circulates along the ring-string, reproducing the known zitterbewegung of the Dirac electron. This pole may be interpreted as a point-like electron, or as a light-like quark confined in the bag.

\section{Conclusion}

The mysterious problem of the source for two-sheeted Kerr geometry leads to a gravitating soliton model, which has to retain the external long-range gravitational and EM field of KN solution. This requires a supersymmetric model of phase transition, in which the Higgs condensate forms a superconducting core of the spinning particle-like solution. The resulting particle has much in common with the famous MIT and SLAC bag models and represents a space-time realization of the Standard Model. Two sheets of the Kerr geometry turn out to be the necessary carriers of the left and right leptons of the SM, in which they are originally massless and get the mass from the Higgs condensate through Yukawa coupling.

Similar to other bag models, the KN bag is pliant to deformations. Spinning bag takes the form of a thin disk, sharp boundary of which represents a ring-string supporting traveling waves. The Compton radius of the bag indicates that it should be associated with a dressed electron. Traveling waves of the ring-string deform the bag creating a singular pole - the circulating end point of the ring-string, which may also be interpreted as a quark confined in the bag. The resulting bag model forms a coherent bag-string-quark complex which unites the dressed and point-like electron, reproducing the known puzzle of zitterbewegung.

\section{References}

[1] Debney G. C., Kerr R. P. and Schild A. 1969 J. Math. Phys. 101842

[2] Burinskii A. 2010 J. Phys. A: Math. Theor. 43392001 [arXiv: 1003.2928].

[3] Burinskii A. 2014 Int J. of Mod. Phys. A 29 1450133, [arXiv:1410.2888].

[4] Burinskii A. 2014, Emergence of the Dirac Equation in the Solitonic Source of the Kerr Spinning Particle, [arXiv:1404.5947].

[5] Chodos A. et al. 1974 Phys. Rev. D 9, 3471

[6] Bardeen W. A. at al. 1974 Phys. Rev. D 11, 1094.

[7] Wess J., Bagger J. Supersymmetry and Supergravity (Princeton Univ. Press, New Jersey), 1983.

[8] Nielsen H. B. and Olesen P. 1973 Nucl. Phys. B 61, 45

[9] Dirac P.A.M. 1962 Proc. R. Soc. Lond. A 268, 57-67.

[10] Burinskii A. 1995 Phys. Rev. D 52 5826, [arXiv:hep-th/9504139].

[11] Burinskii A.Ya. 1974 Sov. Phys. JETP 39193

[12] Ivanenko D.D. and Burinskii A.Ya. 1975 Izv. Vuz. Fiz., 5, 135 